\section{Factores psicosociales asociados a un alto, medio y bajo riesgo de síntomas depresivos en el posparto inmediato}

\author{
Psychosocial factors associated to low, \\ medium and high risk of depressive \\ symptoms in the immediate postpartum \\ period
}

Cynthia Inés Paolini 1

Alicia Juana Oiberman 2

Silvia Balzano 3

\begin{abstract}
Objectives: evaluate psychosocial factors associated to depressive symptoms during the immediate postpartum period.

Methods: a total of 45 argentine puerperal women hospitalized in a public maternal hospital of Buenos Aires, Argentina, were interviewed on the 1st and 3rd day postpartum from March to December 2010. Aiming to assess the presence of depressive symptoms, the Edinburgh Postnatal Depression Scale (EPDS) was administrated. The Perinatal Psychological Interview was administrated to explore psychosocial variables. Based on the scores obtained in the EPDS, the sample was separated in three different groups with increasing risk of depressive symptomatology: "negative or low risk", "positive medium risk" and "positive high risk". Cut-off scores were 9 and 19 points.

Results: out of the total psychosocial variables analyzed, statistically significant differences were found among the three groups regarding family violence, migration, previous history of depression, depressive symptoms during pregnancy and increasing number of life events.

Conclusions: the setting of two cut-off scores in EPDS allows the early detection of women with depressive symptoms. It is suggested the usefulness of cut-off value of 19 for early detection of women at high risk to developing postpartum depression.
\end{abstract}

Key words Depression, Postpartum depression, Early detection
1,2 Centro Interdisciplinario de Investigaciones en Psicología, Matemática y Experimental; Consejo Nacional de Investigaciones Científicas y Técnicas (CIIPME-CONICET). J. D. Perón 2158, Ciudad Autónoma de Buenos Aires, Argentina. E-mail: cynthiapaolini@yahoo.com.ar

3 Consejo Nacional de Investigaciones Científicas y Técnicas (CONICET). Buenos Aires, Argentina.

\title{
Resumen
}

Objetivos: evaluar factores psicosociales asociados a sintomas depresivos en el posparto inmediato.

Métodos: 45 mujeres puérperas de un hospital público de Buenos Aires, Argentina, fueron entrevistadas entre el ler y el 3er día posparto, de marzodiciembre 2010. La Escala de Depresión Posnatal de Edimburgo (EPDS) fue administrada para evaluar sintomas depresivos. La entrevista psicológica perinatal (EPP) fue administrada para detectar variables psicosociales. A partir de los resultados obtenidos en la EPDS, se separó la muestra en tres grupos con diferentes grados de riesgo de sintomatología depresiva: "negativas o de bajo riesgo", "positivas de riesgo medio" y "positivas de alto riesgo". Los puntajes de corte utilizados fueron 9 y 19.

Resultados: se encontraron diferencias significativas entre los tres grupos con violencia familiar, migración, historia previa de depresión, presencia de sintomas depresivos durante el embarazo y mayor cantidad de estresores.

Conclusiones: el establecimiento de los dos puntajes de corte en la EPDS permite una detección temprana de las mujeres que presentan sintomas depresivos. Se sugiere la utilidad del puntaje de corte de 19 para la detección temprana de mujeres con mayor riesgo de desarrollar depresión posparto.

Palabras-clave Depresión, Depresión posparto, Detección precoz 


\section{Introducción}

La prevalencia de depresión posparto reportada en la literatura para países desarrollados es de aproximadamente $10-15 \%$ de las madres, y la prevalencia de "baby blues" puede llegar a ser tan alta como 50-80\%.1-3 Sin embargo, muchos estudios demuestran que la frecuencia difiere no solo entre culturas, sino también entre países de la misma cultura occidental1,4,5 y de acuerdo con los diferentes niveles socioeconómicos, siendo más frecuente para el nivel bajo. 6 Estudios realizados en Chile arrojan una frecuencia de hasta el 50,7\% para depresión posparto. 7,8 Por lo tanto, la prevalencia de depresión posparto fluctúa entre el $8 \%$ y el $50 \%$, siendo tres veces más alta en los países emergentes que en los países desarrollados. 8,9

Los desordenes mentales posparto incluyen el "baby blues", la depresión posparto y la psicosis posparto. El "baby blues" se desarrolla entre el 3er y el 6to día posparto, y puede durar hasta 2 a 6 semanas. Este síndrome está caracterizado por decaimiento anímico, cansancio físico y mental, ansiedad, irritabilidad, llanto y trastornos del sueño. 10

Investigaciones recientes sugieren que las madres en riesgo de desarrollar una depresión posparto pueden ser identificadas tempranamente durante el posparto inmediato como un método de prevención secundaria.11 Se encontraron correlaciones significativas entre el estado de ánimo materno a los 5 días del posparto y a las 6 semanas del posparto.12,13 $\mathrm{Si}$ bien se considera el "baby blues" como un cuadro de menor severidad y duración que la depresión posparto, hay autores que plantean que los síntomas de depresión posparto pueden aparecer tan tempranamente como durante las dos primeras semanas del posparto. ${ }^{14}$ Por otra parte, la presencia de síntomas depresivos en el puerperio inmediato constituye un factor de riesgo para el desarrollo de una posterior depresión posparto, es decir, un "baby blues" severo sería un poderoso predictor de una subsecuente depresión: $40 \%$ de las mujeres con cuadros de "baby blues" severos desarrollaron posteriormente un trastorno depresivo. 15

Tradicionalmente se propone como período de comienzo de la depresión posparto a partir de las 4 a 6 semanas posparto, sin embargo investigaciones recientes en detección precoz plantean que habría evidencia de que la sintomatología depresiva podría aumentar dentro de las dos primeras semanas del posparto y que de las mujeres en riesgo, un porcentaje considerable podría desarrollar depresión posparto durante ese período.11,16 Esto resulta de gran importancia para la detección precoz de este cuadro, teniendo en cuenta que hasta un $50 \%$ de los casos de desórdenes posparto no son diagnosticados ni tratados ${ }^{17}$ y que se trata de una enfermedad que interfiere en el proceso de apego madre-hijo produciendo efectos negativos, tanto para la madre como para el desarrollo del niño si no es tratada a tiempo ${ }^{18} \mathrm{y}$, en general, se encuentra asociada a un severo sufrimiento emocional. 19,20

Un mayor conocimiento acerca de los factores de riesgo que predisponen a las mujeres a presentar tempranamente síntomas depresivos posparto nos permitirá lograr una detección temprana de aquellas que requieren tratamiento preventivo. ${ }^{21}$

\section{Métodos}

El presente estudio es de tipo descriptivo transversal de asociación entre la presencia de síntomas depresivos en el posparto inmediato con variables sociodemográficas, psicosociales, obstétricas y neonatales.

La muestra se encuentra conformada por un total de 45 mujeres puérperas hospitalizadas en sala de internación conjunta del Hospital Materno Infantil "Ana Goitia" de la región Metropolitana de Buenos Aires, Argentina. Dichas mujeres fueron entrevistadas por una de las autoras (CIP) psicóloga perinatal durante el 1 er y el 3 er días posparto, previo al alta, en sala de internación conjunta, desde marzo a diciembre de 2010 .

Las variables analizadas fueron: variables obstétricas - neonatales: sexo del recién nacido $(\mathrm{RN})$, peso del RN (gramos), tipo de parto (normal /cesárea), abortos previos (SI/NO), paridad (primiparidad / paridad: 2-3 hijos / Multiparidad: $>4$ hijos), tipo de alimentación (pecho/mixta/biberón/dificultad de lactancia). Variables socio-demográficas: edad materna y paterna (años), cambio de paternidad (SI/NO), educación materna y paterna (menos de primario completo/primario completo-secundario incompleto/secundario completo-terciario-universitario incompleto/universitario completo), trabajo materno y paterno (SI/NO), situación de pareja(estable conviviendo/estable no conviviendo/ inestable/sola con apoyo familiar/sola sin apoyo familiar), separación durante el embarazo (SI/NO), convivencia a partir del embarazo actual (SI/NO). Variables psicosociales respecto del embarazo actual: embarazo buscado (SI/NO), aceptación del embarazo (SI/NO), percepción del estado físico (se sintió físicamente: Bien/Mal/Regular) y emocional durante el embarazo (se sintió emocionalmente Bien/Mal/Regular), presencia de problemas físicos: 
internación durante el embarazo (SI/NO), hipertensión (SI/NO), pérdidas (SI/NO), infección urinaria (SI/NO), contracciones (SI/NO), diabetes $(\mathrm{SI} / \mathrm{NO})$, embarazo como "life event (SI/NO)", parto como "life event (SI/NO)". Problemas emocionales durante el embarazo: temor por la salud del bebé (SI/NO), temor por la salud propia (SI/NO), angustia y ansiedad (SI/NO), temor al parto (SI/NO). Conflictos infantiles de la madre: abandono de alguno de los padres (SI/NO), fallecimiento de alguno de los padres (SI/NO), maltrato (SI/NO). Soporte familiar (SI/NO). Soporte de la pareja (SI/NO).

Se indagaron todos los estresores durante el embarazo o en años anteriores propuestos por la entrevista psicológica perinatal (EPP).22 Se incluyeron algunas otras variables específicas del tema de investigación: historia previa de depresión posparto (SI/NO) y presencia de síntomas depresivos durante el embarazo (SI/NO). Todos los datos se obtuvieron a partir de lo referido por la madre durante la entrevista. Como así también los estresores se indagaran a partir de lo emergente en la entrevista. La variable cambio de paternidad se refiere a los casos en que la mujer tiene algún hijo de otro padre diferente de la pareja actual. La variable presencia de síntomas depresivos durante el embarazo fue indagada retrospectivamente a partir de lo referido por la madre en casos de presencia de síntomas como: llanto acompañado de angustia, inestabilidad emocional, angustia incrementada a partir del último trimestre (referidos con angustia al momento de la entrevista), pérdida del apetito, ansiedad, tendencia al encierro y aislamiento.

En la investigación se utilizó la Escala de Depresión Posnatal de Edimburgo (EPDS). ${ }^{23}$ Para evaluar la asociación entre la variable dependiente (puntaje final de la EPDS) y las variables independientes analizadas, los individuos fueron clasificados en tres grupos a partir de la utilización de 2 puntos de corte dentro de la escala EPDS, a saber: (1) "negativos" o de "bajo riesgo" con un puntaje menor a 9; (2) "positivos de riesgo medio" con un puntaje de 9 a 18 puntos; y (3) "positivos de alto riesgo" con un puntaje mayor o igual a 19 puntos.

El primer punto de corte de 9 puntos está basado en lo reportado por los autores de la escala EPDS quienes sugieren este valor de puntaje de corte para cuando esta escala es utilizada como método de screening, siendo negativos los casos con puntajes por debajo de los 9 puntos y positivos los casos que obtuvieron desde 9 a 30 puntos. 23 Este punto de corte coincidió además con el percentil 50 de la distribución total de valores de EPDS observada en la presente muestra.

Se definió un segundo punto de corte en un valor de 19 puntos, que corresponde al percentil 75 de la distribución de valores de EPDS en el total de individuos de la muestra con el fin de describir las características de un potencial grupo de "positivos de alto riesgo" correspondiente al cuartil con mayor puntaje obtenido.

Para determinar asociaciones con los resultados obtenidos en la EPDS, se utilizaron diferentes tests estadísticos de acuerdo con la variable evaluada. Se utilizó el $\chi^{2}$ o test exacto de Fisher para variables categóricas y ANOVA o test de medianas en casos de variables continuas. Las asociaciones con un valor de $p<0.05$ fueron consideradas estadísticamente significativas.

Para las variables que mostraron diferencias estadísticamente significativas entre los tres grupos se evaluó mediante un test de tendencia noparamétrico entre grupos ordinales, la existencia de un aumento o disminución de la variable evaluada entre las 3 categorías de valores de la EPDS ("negativas o de bajo riesgo", "positiva de riesgo medio" y "positivas de alto riesgo").

Para todos los análisis estadísticos se utilizó el STATA 8.0 statistical software package (Statistics/ Data Analysis 8.0, Stata Corporation 4905 Lakeway Drive College Station, Texas 77845 USA. http://www.stata.com).

\section{Resultados}

En la Tabla 1 se exponen las características neonatales, obstétricas y socio-demográficas de la muestra analizada. Se observó una media de edad materna de 26.8 y paterna de 29.9 años.

Con respecto al nivel de educación se observa un nivel similar entre las madres y los padres. Ninguno de los individuos tiene estudios universitarios completos. El $26.3 \%$ de los padres y el $34.1 \%$ de las madres finalizaron los estudios secundarios. El $65.8 \%$ de los padres y el $56.8 \%$ de las madres tiene estudios primarios completos o secundarios incompletos. El $7.9 \%$ de los padres y el $9.1 \%$ de las madres no finalizaron los estudios primarios. Existen diferencias respecto del trabajo entre los padres y las madres de la muestra. Es mucho mas frecuente el trabajo paterno $(86.7 \%)$ que el trabajo materno $(22.2 \%)$. Con respecto a la situación de la pareja se observa que la mayoría de las parejas de la muestra (73.3\%) son parejas estables que conviven juntos, de los cuales el $11.1 \%$ conviven a partir de este embarazo actual. El $6.8 \%$ son parejas también estables pero no conviviendo al momento en que se 
realizó el estudio y el 2.2\% son parejas inestables. $13.3 \%$ de las mujeres entrevistadas no tienen pareja pero cuentan con apoyo de su familia y el $2.2 \%$ están sin pareja y sin apoyo familiar. Del total de las mujeres entrevistadas hay un $15.6 \%$ que se han separado durante el embarazo. El $46.7 \%$ de las madres tiene hijos con otras parejas es decir hubo cambio de paternidad (Tabla 1).

La Tabla 2 resume las variables psicosociales indagadas respecto del embazo actual. Se observa que el $48.9 \%$ de la muestra buscó el embarazo actual. El $77.7 \%$ aceptó inmediatamente el embarazo, el $6.7 \%$ no aceptó el embarazo durante toda la duración del mismo y el $15.6 \%$ no lo aceptó al principio el embarazo. El $57.8 \%$ de las mujeres entrevistadas dicen haberse sentido bien físicamente durante el embarazo mientras que el $28.0 \%$ dice haberse sentido mal y el $8.9 \%$ regular. Con respecto a la percepción del estado emocional durante el embarazo, el $48.9 \%$ refiere haberse sentido bien emocionalmente, el $31.1 \%$ mal de las cuales lo más frecuente ha sido la presencia de estados de angustia y ansiedad durante el embarazo en un 33,3\% de los casos. Respecto de los problemas físicos durante el embarazo lo mas frecuente fueron las contracciones durante el embarazo en un $15.6 \%$, solo un $6.7 \%$ de la muestra fueron internadas durante el embarazo por la presencia de algún problema físico.

En la Tabla 3 se exponen los resultados del análisis de las variables estudiadas para los tres grupos: "negativas o de bajo riesgo", "positivas de riesgo medio" y "positivas de alto riesgo" de acuerdo al puntaje obtenido en la EPDS. La prevalencia para el grupo de negativas de bajo riesgo fue: $24,4 \%(\mathrm{n}=11)$, positivas de riesgo medio: $48,9 \%$ $(n=22)$ y positivas de alto riesgo: $26,7 \%(n=12)$. Del total de variables psicosociales analizadas, se encontraron diferencias estadísticamente significativas entre los tres grupos para: violencia familiar, migración, historia previa de depresión, presencia de síntomas depresivos durante el embarazo y cantidad de estresores ("life events") en el último año y en años anteriores.

La Tabla 4 muestra los resultados obtenidos a partir de los test de tendencia. Se observó una tendencia estadísticamente significativa entre mayores puntajes en la EPDS con: mayor cantidad de estresores ("life events"); aumento de la frecuencia de antecedentes de depresión; mayor frecuencia de síntomas depresivos durante el embarazo y violencia familiar.

Tabla 1

Características socio-demográficas de la muestra $(\mathrm{N}=45)$.

\section{Características de los recién nacidos}

$\begin{array}{lc}\text { Sexo }-\mathrm{n}(\%) & \\ \text { Femenino } & 18(40.0) \\ \text { Masculino } & 27(60.0) \\ \text { Peso }(\mathrm{g}) & \\ \overline{\mathrm{X}} \pm \mathrm{DS} & 3316 \pm 485 \\ \mathrm{~min}-\mathrm{max} & 2150-4900 \\ \text { Tipo de parto - } \mathrm{n}(\%) & \\ \quad \text { Cesárea } & 7(15.6) \\ \text { Parto normal } & 38(84.4) \\ \text { Alimentación - } \mathrm{n}(\%) & \\ \text { Pecho } & 38(84.4) \\ \text { Mixta } & 3(6.7) \\ \text { Biberón } & 1(2.2) \\ \text { Dificultad de lactancia } & 3(6.6)\end{array}$


Características socio-demográficas de la muestra $(\mathrm{N}=45)$.

\section{Características maternas y paternas}

\begin{tabular}{|c|c|}
\hline \multicolumn{2}{|l|}{$\operatorname{Edad}(\bar{X} \pm D S)$} \\
\hline Materna & $26.8 \pm 7.3$ \\
\hline Paterna & $29.9 \pm 9.1$ \\
\hline Paridad - mediana $\left(\mathrm{P}_{25}-\mathrm{P}_{75}\right)$ & $2(1-4)$ \\
\hline Primiparidad $(n-\%)$ & $15(33.3)$ \\
\hline Paridad: $2-3$ hijos & $18(40.0)$ \\
\hline Multiparidad: 4 o + hijos ( $n-\%)$ & $12(26.67)$ \\
\hline Cambio de paternidad $(n-\%) *$ & $14(46.7)$ \\
\hline Abortos previos ( $n-\%)$ & $5(11.1)$ \\
\hline \multicolumn{2}{|l|}{ Educación - n (\%) } \\
\hline \multicolumn{2}{|l|}{ Educación materna $\mathrm{n}(\%) * *$} \\
\hline Menor de primario completo & $4(9.1)$ \\
\hline Primario completo - Secundario incompleto & $25(56.8)$ \\
\hline Sec. completo - Terciario - Univ. incompleto & $15(34.1)$ \\
\hline Universitario completo & $0(0.0)$ \\
\hline \multicolumn{2}{|l|}{ Educación paterna $(n) * * *$} \\
\hline Menor de primario completo & $3(7.9)$ \\
\hline Primario completo - Secundario incompleto & $25(65.8)$ \\
\hline Sec. completo - Terciario - Univ. incompleto & $10(26.3)$ \\
\hline Universitario completo & $0(0.0)$ \\
\hline \multicolumn{2}{|l|}{ Trabajo - n (\%) } \\
\hline Materno & $10(22.2)$ \\
\hline Paterno & $39(86.67)$ \\
\hline \multicolumn{2}{|l|}{ Situación de pareja - n (\%) } \\
\hline Pareja estable conviviendo & $33(73.3)$ \\
\hline Pareja estable no conviviendo & $3(6.8)$ \\
\hline Pareja inestable & $1(2.2)$ \\
\hline Sin pareja con apoyo familiar & $6(13.3)$ \\
\hline Sin pareja sin apoyo familiar & $1(2.2)$ \\
\hline Separación durante el embarazo - n (\%) & $7(15.6)$ \\
\hline Convivencia a partir del embarazo actual - n (\%) & $5(11.1)$ \\
\hline
\end{tabular}

* $N^{\circ}$ de no especificados: $3 ; * * N^{\circ}$ de no especificados: $1 ; * * * N^{\circ}$ de no especificados: 7 . 
Variables psicosociales respecto del embarazo actual $(n=45)$.

\begin{tabular}{|c|c|c|}
\hline Embarazo actual & $\mathbf{N}$ & $\%$ \\
\hline Embarazo buscado & 22 & 48.9 \\
\hline \multicolumn{3}{|l|}{ Aceptación del embarazo } \\
\hline Sí & 35 & 77.7 \\
\hline No & 3 & 6.7 \\
\hline Al principio no aceptó & 7 & 15.6 \\
\hline \multicolumn{3}{|l|}{ Como se sintió físicamente* } \\
\hline Bien & 26 & 57.8 \\
\hline Mal & 13 & 28.9 \\
\hline Regular & 4 & 8.9 \\
\hline \multicolumn{3}{|l|}{ Como se sintió emocionalmente** } \\
\hline Bien & 22 & 48.9 \\
\hline Mal & 14 & 31.1 \\
\hline Regular & 7 & 15.6 \\
\hline \multicolumn{3}{|l|}{ Problemas físicos durante el embarazo } \\
\hline Internación durante el embarazo & 3 & 6.7 \\
\hline Hipertensión & 2 & 4.4 \\
\hline Pérdidas & 4 & 8.9 \\
\hline Infección urinaria & 3 & 6.7 \\
\hline Contracciones & 7 & 15.6 \\
\hline Diabetes & 1 & 2.2 \\
\hline Embarazo como "life event" & 11 & 24.4 \\
\hline Parto como "life event" & 5 & 11.1 \\
\hline \multicolumn{3}{|l|}{ Problemas emocionales durante el embarazo } \\
\hline Temor por la salud del bebé & 13 & 28.9 \\
\hline Temor por la salud propia & 2 & 4.4 \\
\hline Angustia y ansiedad & 15 & 33.3 \\
\hline Temor al parto & 6 & 13.3 \\
\hline \multicolumn{3}{|l|}{ Conflictos infantiles de la madre } \\
\hline Abandono de alguno de los padres & 6 & 13.3 \\
\hline Fallecimiento de alguno de los padres & 2 & 4.4 \\
\hline Maltrato & 5 & 11.1 \\
\hline
\end{tabular}

* $\mathrm{N}^{\circ}$ de no especificados: $2 ; * * \mathrm{~N}^{\circ}$ de no especificados: 2 . 
Características psicosociales según resultado en la Escala de Depresión Posnatal de Edimburgo (EPDS) (N=45).

\begin{tabular}{|c|c|c|c|c|}
\hline \multirow{2}{*}{$\begin{array}{l}\text { Variables socio-demográficas } \\
\text { y psicológicas }\end{array}$} & \multicolumn{3}{|c|}{ Resultado en EPDS } & \multirow[b]{2}{*}{$p^{1}$} \\
\hline & $\begin{array}{c}\text { "Negativas bajo riesgo" } \\
\text { (0-8 puntos) } \\
\mathrm{N}=11(24.4 \%)\end{array}$ & $\begin{array}{c}\text { "Positivas riesgo medio" } \\
\text { (9-18 puntos) } \\
\mathrm{N}=22(48.9 \%)\end{array}$ & $\begin{array}{l}\text { "Positivas alto riesgo" } \\
\text { (19-30 puntos) } \\
\mathrm{N}=12(26.7 \%)\end{array}$ & \\
\hline Sexo recién nacido - $\mathrm{n}(\%)$ & & & & 0.450 \\
\hline Femenino & $6(54.5)$ & $7(31.8)$ & $5(41.7)$ & \\
\hline Masculino & $5(45.5)$ & $15(68.2)$ & $7(58.3)$ & \\
\hline Peso recién nacido & & & & 0.682 \\
\hline $\bar{X} \pm D S$ & $3426(571)$ & $3267(445)$ & $3297(496)$ & \\
\hline Tipo de parto - n (\%) & & & & 0.430 \\
\hline Cesárea & $3(27.3)$ & $3(13.6)$ & $1(8.3)$ & \\
\hline Parto normal & $8(72.7)$ & $19(86.4)$ & $11(91.7)$ & \\
\hline Alimentación - n (\%) & & & & 0.516 \\
\hline Pecho & $10(90.9)$ & $20(90.9)$ & $9(75.0)$ & \\
\hline Biberón/Mixta/Dificultad de lactancia & $1(9.09)$ & $2(9.09)$ & $3(25.0)$ & \\
\hline \multicolumn{5}{|l|}{ Edad (mediana $\mathrm{P}_{25}-\mathrm{P}_{75}$ ) } \\
\hline Materna & $26(21-29)$ & $26.5(20-31)$ & $26(22.5-34)$ & 0.733 \\
\hline Paterna & $23(22-31)$ & $30(24-36)$ & $30(23-31)$ & 0.837 \\
\hline Paridad (mediana $\mathrm{P}_{25}-\mathrm{P}_{75}$ ) & $2(1-3)$ & $2(1-4)$ & $2(1.5-4.5)$ & 0.598 \\
\hline Multiparidad: $40+$ hijos ( $n-\%)$ & $1(9.1)$ & $7(31.8)$ & $4(33.3)$ & 0.346 \\
\hline Primiparidad $(n-\%)$ & $4(36.4)$ & $8(36.4)$ & $3(25.0)$ & 0.843 \\
\hline Cambio de paternidad $(\mathrm{n}-\%)^{1}$ & $3(42.9)$ & $6(42.9)$ & $5(55.6)$ & 0.896 \\
\hline Abortos previos $(n-\%)$ & $2(18.2)$ & $1(4.5)$ & $2(16.7)$ & 0.281 \\
\hline \multicolumn{5}{|l|}{ Educación - n (\%) } \\
\hline \multicolumn{5}{|l|}{ Educación materna $(n)^{2}$} \\
\hline Menor de primario completo & $1(9.1)$ & $1(4.5)$ & $2(18.2)$ & \\
\hline Prim. completo - Sec. incompleto & $4(36.4)$ & $15(68.2)$ & $6(54.5)$ & \\
\hline Sec. comp. - Terciario - Univ. incom. & $6(54.5)$ & $6(27.3)$ & $3(27.3)$ & 0.306 \\
\hline \multicolumn{5}{|l|}{ Educación paterna $(n)^{3}$} \\
\hline Menor de primario completo & $0(0.0)$ & $2(9.5)$ & $1(12.5)$ & \\
\hline Prim. completo - Sec. incompleto & $5(55.6)$ & $15(71.4)$ & $5(62.5)$ & \\
\hline Sec. comp. - Terciario - Univ. incom. & $4(44.4)$ & $4(19.0)$ & $2(25.0)$ & 0.585 \\
\hline \multicolumn{5}{|l|}{ Trabajo - n (\%) } \\
\hline Materno & $2(18.2)$ & $4(18.2)$ & $4(33.3)$ & 0.557 \\
\hline Paterno & $8(72.7)$ & $20(95.2)$ & $11(91.7)$ & 0.151 \\
\hline \multicolumn{5}{|l|}{ Situación de pareja - n (\%) } \\
\hline Con pareja & $9(81.8)$ & $19(86.4)$ & $8(66.7)$ & \\
\hline Pareja inestable o sin pareja & $2(18.2)$ & $3(13.6)$ & $4(33.3)$ & 0.392 \\
\hline Separación durante el embarazo - n (\%) & $2(18.2)$ & $2(9.1)$ & $3(25.00)$ & 0.427 \\
\hline Convivencia a partir embarazo actual $-\mathrm{n}(\%)$ & $1(9.1)$ & $2(9.1)$ & $2(16.67)$ & 0.834 \\
\hline Embarazo buscado / planificado - $\mathrm{n}(\%)$ & $6(54.4)$ & $11(50.0)$ & $5(41.7)$ & 0.818 \\
\hline \multicolumn{5}{|l|}{ Tipo de life events } \\
\hline Violencia familiar - n (\%) & $0(0.0)$ & $5(22.7)$ & $6(50.0)$ & 0.015 \\
\hline Desarraigo / migración - $\mathrm{n}(\%)$ & $2(18.2)$ & $0(0.0)$ & $3(25.0)$ & 0.042 \\
\hline Peleas con la pareja - n (\%) & $0(0.0)$ & $2(9.1)$ & $4(33.3)$ & 0.059 \\
\hline Cantidad de "life events" $-\mathrm{P}_{50}\left(\mathrm{P}_{25}-\mathrm{P}_{75}\right)$ & $1(0-3)$ & $3(1-4)$ & $5(3-7.5)$ & 0.043 \\
\hline Menor percepción de apoyo de la pareja $4-n(\%)$ & $2(22.2)$ & $3(15.8)$ & $4(36.4)$ & 0.490 \\
\hline Menor percepción de apoyo de flia propia 5 - n (\%) & $1(11.1)$ & $3(17.6)$ & $2(20.0)$ & 1.000 \\
\hline Antecedentes personales de depresión - n (\%) & $0(0.0)$ & $6(30.0)$ & $7(70.0)$ & 0.002 \\
\hline Síntomas depresivos durante el embarazo - n (\%) & $0(0.0)$ & $7(36.8)$ & $10(90.9)$ & 0.001 \\
\hline
\end{tabular}

1 Valor de $p$ correspondiente a la prueba de Ji-Cuadrado, Test Exacto de Fisher o Test de Medianas; $2 \mathrm{~N}^{\circ}$ de no especificados: $1 ; 3 \mathrm{~N}^{\circ}$ de no especificados: $7 ;{ }^{\circ} \mathrm{N}^{\circ}$ de no especificados: $6 ; 5 \mathrm{~N}^{\circ}$ de no especificados: 9. 


\begin{tabular}{|c|c|c|c|c|c|}
\hline \multirow[b]{2}{*}{ Variables } & \multicolumn{3}{|c|}{ Resultado en la EPDS } & \multicolumn{2}{|c|}{ Test de Tendencia } \\
\hline & $\begin{array}{l}\text { "Negativas bajo } \\
\text { riesgo" } \\
(0-8 \text { puntos }) \\
\text { N=11(24.4\%) }\end{array}$ & $\begin{array}{c}\text { "Positivas riesgo } \\
\text { medio" } \\
\text { (9-18 puntos) } \\
\mathrm{N}=22(48.9 \%)\end{array}$ & $\begin{array}{c}\text { "Positivas alto } \\
\text { riesgo" } \\
\text { (19-30 puntos) } \\
\mathrm{N}=12(26.7 \%)\end{array}$ & Valor1 & $p$ \\
\hline Cantidad de "life events" $P_{50}\left(P_{25}-P_{75}\right)$ & $1(0-3)$ & $3(1-4)$ & $5(3-7.5)$ & $\mathrm{Z}=3.3$ & 0.001 \\
\hline Antecedentes personales de depresión - n (\%) & $0(0.0)$ & $6(30.0)$ & $7(70.0)$ & $\chi^{2}=9.3$ & 0.009 \\
\hline Síntomas depresivos durante el embarazo - $n(\%)$ & $0(0.0)$ & $7(36.8)$ & $10(90.9)$ & $\chi^{2}=16.8$ & 0.001 \\
\hline Violencia familiar - n (\%) & $0(0.0)$ & $5(22.7)$ & $6(50.0)$ & $\chi^{2}=7.6$ & 0.006 \\
\hline Desarraigo / migración - n (\%) & $2(18.2)$ & $0(0.0)$ & $3(25.0)$ & $\chi^{2}=0.3$ & 0.560 \\
\hline
\end{tabular}

EPDS = Escala de Depresión Posnatal de Edimburgo; 1 El valor de Z fue obtenido mediante un test de tendencia para grupos ordinales; el valor de $\chi^{2}$ fue obtenido mediante un Ji-cuadrado de tendencia. En todos los casos se consideró un nivel de significancia del 0.05 .

\section{Discusión}

Ambos puntos de corte aplicados nos permiten diferenciar no sólo a aquellas mujeres con alto riesgo de sintomatología depresiva en el posparto inmediato, sino también a las que serían más propensas a presentar síntomas depresivos en etapas posteriores posparto inmediato.

Ha sido reportado previamente que hasta el 50\% de las mujeres con algún desorden psiquiátrico en el posparto presentarían síntomas depresivos durante el embarazo. 24 Este dato concuerda con los resultados hallados en esta investigación, en donde se advierte que el $36.8 \%$ de las mujeres con puntajes por encima de 9 puntos en la EPDS, así como el 90\% de las que obtuvieron puntajes por encima de 19 puntos, es decir, las que pertenecían al grupo de alto riesgo, habría presentado síntomas depresivos durante el embarazo versus un $0.0 \%$ en el grupo de las mujeres sin síntomas depresivos. Estos hallazgos coinciden con O'Hara et al. ${ }^{25}$ quien ha reportado que la presencia de síntomas depresivos en el embarazo podría predecir el comienzo de un "baby blues". El estado de ánimo durante el tercer trimestre predice la subsecuente sintomatología depresiva en el período del posparto temprano. ${ }^{14}$ Estos datos sugieren que un

\section{Referencias}

1. O'Hara MW, Swain AM. Rates and risk of postpartum depression-a meta-analysis. Int Rev Psychiatry. 1996; 8: $37-54$. gran porcentaje de las mujeres que presentan síntomas depresivos en el posparto inmediato presentarían algunos de estos síntomas durante el embarazo, lo que resulta de gran importancia ya que justifica la detección temprana de mujeres en riesgo durante el embarazo como método de prevención.

Así mismo, debe mencionarse que los factores de riesgo asociados al grupo de mayor riesgo de sintomatología depresiva posparto en este estudio concuerdan con los reportados en la bibliografía existente: historia personal de desordenes afectivos, 26,27 historia previa de depresión posparto en partos anteriores 28 han sido todos factores de riesgo consistentemente estudiados y encontrados como importantes factores de riesgo para la depresión posparto. Del mismo modo, ha quedado demostrado que la presencia de un "baby blues" severo sería un poderoso predictor de una subsecuente depresión: $40 \%$ de las mujeres con cuadros de "baby blues" severos desarrollarían posteriormente un trastorno depresivo. ${ }^{29}$ Esto sugiere que aquellas mujeres que conforman el tercer grupo, de alto riesgo de síntomas depresivos en el posparto inmediato estarían en mayor riesgo de continuar con una depresión posparto.
2. Kendell RE. Emotional and physical factors in the genesis of puerperal mental disorders. J Psychosom Res. 1985; 29: 3-11. 
3. Horowitz JA, Goodman JH. Identifying and treating postpartum depression. J Obstet Gynecol Neonatal Nurs. 2005; 34: 264-73.

4. Romito P, Saurel-Cubizolles MJ, Lelong N. What makes new mothers unhappy: psychological distress one year after birth in Italy and France. Soc Sci Med. 1999; 49: 1651-61.

5. Forman DN, Videbech P, Hedegaard M, Dalby Salving J, Secher NJ. Postpartum depression: identification of women at risk. Br J Obstet Gynaecol. 2000; 107: 1210-7.

6. Segre LS, O'Hara MW, Arndt S, Stuart S. The prevalence of postpartum depression: the relative significance of three social status indices. Soc Psychiatr Psychiatr Epidemiol. 2007; 42: 316-21.

7. Jadresic E, Araya R. Prevalencia de depresión posparto y factores asociados en Santiago, Chile. Rev Méd Chile. 1995; 123: 694-9.

8. Póo AM, Espejo C, Godoy C, Gualda de la M, Hernández T, Pérez C. Prevalencia y factores de riesgo asociados a la depresión posparto en puérperas de un Centro de Atención Primaria en el Sur de Chile. Rev Med Chile. 2008; 136: 44 52

9. Rojas G, Fritsch R, Guajardo V, Rojas F, Barroilhet S, Jadresic E. Caracterización de madres deprimidas en el posparto. Rev Med Chile. 2010; 138: 536-42.

10. Koshchavtsev AG, Mul'tanovskaya VN, Lorer VV. Baby blues syndrome as an adaptation disorder in the early stages of formation of the mother-child system. Neurosci Behav Physiol. 2008; 38: 439-42.

11. Dennis CL. Can we identify mothers at risk for postpartum depression in the immediate postpartum period using the Edinburgh Postnatal Depression Scale? J Affect Disord. 2004; 78: 163-9

12. Hannah P, Adams D, Lee A, Glover V, Sandler M. Links between early post-partum mood and post-natal depression. Br J Psychiatr. 1992; 160: 777-80.

13. Yamashita H, Yoshida K, Nakano H, Tashiro N. Postnatal depression in Japanese women: detecting the early onset of postnatal depression by closely monitoring the postpartum mood. J Affect Disord. 2000; 58: 145-54.

14. Bloch M, Rotenberg N, Koren D, Klein E. Risk factors for early postpartum depressive symptoms. Gen Hosp Psychiatr. 2006; 28: 3-8.

15. Henshaw C. Mood disturbance in the early puerperium: review. Arch Womens Ment Health. 2003; 6: 33-42.
16. Wisner KL, Perel JM, Peindl KS, Hanusa BH. Timing of depression recurrence in the first year after birth. J Affect Disord. 2004; 78: 249-52.

17. Ramsay R. Postnatal depression. Lancet. 1993; 341: 1358.

18. Murray L. The impact of postnatal depression on infant development. J Child Psychol Psychiatr. 1992; 33: 543-61.

19. Kumar R, Robson KM. A prospective study of emotional disorders in childbearing women. Br J Psychiatr. 1984; 144 $35-47$.

20. Stowe ZN, Nemeroff CH. Women at risk for postpartumonset major depression. Am J Obstet Gynecol. 1995; 173: 639-45.

21. Wisner KL, Wheeler SB. Prevention of recurrent postpartum major depression. Hosp Community Psychiatry. 1994; 45: 1191-6.

22. Oiberman AJ. Un nuevo instrumento diagnóstico en situaciones perinatales: la entrevista psicológica de diseño óptico. Rev Investig Psicol (On line). 2000; 5: 77-89.

23. Cox JL, Holden JM, Sagovsky R. Detection of postnatal depression: development of the 10-item Edinburgh Postnatal Depression Scale. Br J Psychiatr. 1987; 150: 782-

24. Dean C, Williams RJ, Brockington IF. Is puerperal psychosis the same as bipolar manic-depressive disorder? A family study. Psychol Med. 1989; 19: 637-47.

25. O'Hara MW, Schlechte JA, Lewis DA, Varner MW. Controlled prospective study of postpartum mood disorders: psychological, environmental, and hormonal variables. J Abnorm Psychol. 1991; 100: 63-73.

26. Schaper AM, Rooney BL, Kay NR, Silva PD. Use of the Edinburgh Postnatal Depression Scale to identify postpartum depression in a clinical setting. J Reprod Med. 1994; 39: 620-4.

27. Garvey MJ, Tuason VB, Lumry AE, Hoffmann NG Occurrence of depression in the postpartum state. J Affect Disord. 1983; 5: 97-101.

28. Bergant AM, Heim K, Ulmer H, Illmensee K. Early postnatal depressive mood: associations with obstetric and psychosocial factors. J Psychosom Res. 1999; 46: 391-4.

29. Henshaw C. Clinical and biological aspects of postpartum blues and depression. Curr Opin Psychiatr. 2000, 13: 635-8.

Recebido em 13 de setembro de 2011

Versão final apresentada em 16 de janeiro de 2012

Aprovado em 24 de fevereiro de 2012 